\title{
POLÍTICA Y POLÍTICAS SOCIALES EN EL ECUADOR RECIENTE: DIFICULTADES ASOCIADAS A LA SALIDA DEL CICLO NEOLIBERAL
}

\section{POLITICAL AND SOCIAL POLICIES IN ECUADOR RECENT: PROBLEMS ASSOCIATED WITH THE DEPARTURE OF THE CYCLE NEOLIBERAL}

\author{
Analía Minteguiaga*
}

RESUMEN

Partiendo de la idea de que el proyecto neoliberal operó una contracción y tránsito de la política al ámbito de la "sociedad civil", que supuso desafectar al Estado y trasladar la discusión por las condiciones materiales de vida al mercado, el artículo analiza lo sucedido en Ecuador en las últimas décadas, haciendo énfasis en el campo de las políticas sociales y exponiendo algunas dificultades asociadas al desarme de aquella operatoria.

PALABRAS CLAVE: ECUADOR * NEOLIBERALISMO * POLÍTICA SOCIAL * CONDICIONES DE VIDA * REVOLUCIÓN CIUDADANA

\section{ABSTRACT}

Based on the idea that the neoliberal project operated a contraction and transit policy within the scope of "civil society", which involved disaffect the State and move the discussion about the material conditions of life to the market, the article examines what happened in Ecuador in recent decades, with emphasis on the social policies field and discusses some difficulties associated with the disarmament of this operative.

KEYWORDS: ECUADOR * NEOLIBERALISM * SOCIAL POLICY * LIFE CONDITIONS * CITIZEN REVOLUTION

Grupo de Estudio sobre Políticas Sociales $y$ Condiciones de Trabajo, Instituto de Investigaciones Gino Germani, Universidad de
Buenos Aires, Argentina (UBA) y además, en el Instituto de Altos Estudios Nacionales del Ecuador. analiaminte@hotmail.com 


\section{INTRODUCCIÓN}

Múltiples trabajos académicos han tenido por objeto de estudio las políticas sociales en América Latina, durante la etapa neoliberal. Dentro de este conjunto de aportes, algunos dieron un paso más allá y vincularon tales intervenciones del Estado con la producción de un nuevo orden como totalidad, es decir, con la instauración de una nueva pauta de relación entre Estado, sociedad y mercado (Danani y Hintze, 2011: 10; Danani y Grassi, 2008: 266). Esto a partir de una conceptualización amplia y compleja de las políticas sociales, en tanto "organizadoras de la vida social e individual" (Danani y Grassi, 2008: 259). Bajo esta mirada, el presente escrito busca problematizar uno de los aspectos que involucró el orden neoliberal. Específicamente aquel que planteó los límites, márgenes y espacios de la disputa por las condiciones materiales de vida y que por ende, tuvo efectos profundos en la manera de concebir las intervenciones sociales del Estado.

La propuesta del artículo adquiere relevancia a la luz de una serie de transformaciones que estarían dando evidencia de la inauguración de un nuevo "ciclo histórico" (Danani y Beccaria, 2011: 106), en el marco de la instauración de gobiernos que se autoproclaman o que son denominados - por sus defensores como detractores - como de "izquierda" o "progresistas". Al menos discursivamente y más evidentemente, bajo algunos cambios de carácter institucional, se observan esfuerzos por marcar un rumbo distinto, por modificar aquel estado de cosas que caracterizó al neoliberalismo dominante de los noventa. Este ciclo - como todos - se erige y justifica a partir de la confrontación, de las "distancias y quiebres", respecto al precedente. Desde este lugar interesa rastrear lo sucedido en Ecuador, específicamente en el campo de las políticas sociales, a partir de la llegada al poder de Rafael Correa y el proceso de cambio por él liderado, conocido oficialmente como "Revolución Ciudadana". En este sentido, la posibilidad de capturar qué elementos "nuevos, viejos o remozados" trajo aparejados el proceso ecuatoriano, estará atada a la caracterización propuesta del ciclo antecedente. Es decir, a aquel aspecto antes mencionado y a sus consecuencias sobre el campo de las políticas sociales. De esta forma, el texto no solo intenta resguardarse de caer en una suerte de descripción ficcional, sin orden lógico ni sentido, sino en la tentación, como indican Danani y Grassi, de quedar atrapados en el "conformismo" bajo el cual todo cambio se constituye per se en "positivo" y/o "progresista" o en el "oposicionismo", en donde, únicamente se registra lo que falta, la insuficiencia y la continuidad (2008: 261). El mejor antídoto ante tales males no solo es mantener una mirada histórica ajustada al recorte propuesto de la realidad (problematización), sino una perspectiva de procesos, ya que solamente en ellos se pueden capturar las transformaciones (que siempre contienen algo del pasado), las "contradicciones (en su sentido productivo de que ellas son puntos de apoyo para nuevas transformaciones), [y] preguntarnos por los vacíos" (2008: 261).

El artículo está integrado por tres secciones. La primera, se adentra en el caso ecuatoriano e incluye la descripción analítica de lo acontecido en el "ciclo histórico" neoliberal (1982-2006) conectando la productividad de aquel componente en el ámbito de las políticas sociales. La segunda, analiza lo sucedido en el periodo de la "Revolución Ciudadana" (2007-2011) y esboza una serie de dificultades asociadas al desarme de dicho ciclo. La tercera, contiene algunas consideraciones finales.

\section{ECUADOR: NEOLIBERALISMO Y POLÍTICAS SOCIALES (1982-2006)}

Como fue desarrollado ampliamente por la literatura especializada (entre otros: Lechner, 1982 y Offe, 1996), el neoliberalismo contiene un componente neoconservador que llevó a poner entre paréntesis la disputa por las condiciones materiales de vida y redefinió el papel del Estado y sus intervenciones (incluyendo las de carácter social). Esa disputa se trasladó a la "sociedad civil", trasmutada a su vez, en un orden mercantil, por lo que la responsabilidad colectiva por la vida del conjunto de miembros de una sociedad devino en responsabilidad de cada uno.

No obstante, en el caso concreto, el ciclo neoliberal asumió especificidades. En Ecuador, 
este traslado de la política a la sociedad civil y con ella, a la "puesta en caja" de las disputas por las condiciones materiales de vida y los esfuerzos por la "socialización de la vida", si bien logró concretarse, tuvo algunos tintes únicos que vale la pena mencionar. El primero, refiere al "grado" de reducción de la política que se debió operar. Esto se vincula, entre otros elementos, con el nivel de desarrollo e institucionalización que tuvo la formación estatal previa al ciclo neoliberal en clave de "intervención de la economía" y "bienestarista" en el campo social. El segundo, refiere a los límites locales que tuvieron las alianzas socioeconómicas para instrumentar algunas políticas neoliberales $y$ derivado de ello, en tercer lugar, la generación $y$ despliegue de espacios en la sociedad civil que pudieron resistir la lógica mercantil y disputar políticamente, aunque sea bajo formas marginales y reactivas. Todas estas particularidades, sin embargo, no cuestionarán la hegemonía del neoliberalismo en el país.

\section{a) LOS PROLEGÓMENOS DEL NEOLIBERALISMO Y SUS PRIMEROS AVANCES (1970-1980)}

La etapa previa al advenimiento neoliberal en Ecuador, daba cuenta de un Estado y de amplios espacios de la vida social bajo una lógica "no regulada". Se trató de una formación estatal y una economía que incorporó ciertos elementos del modelo desarrollista $y$ un conjunto de políticas que podrían denominarse proto "bienestaristas". El rezago puede observarse de hecho, en el inicio y duración de dicha etapa. En este sentido, existe acuerdo en sostener que en Ecuador comienza recién en 1960, cuando tardíamente se intenta una tibia industrialización por sustitución de importaciones y que es en 1970, con el boom petrolero, que se llevan adelante acciones públicas de intervención en la economía, procesos de reforma agraria y relativa expansión de los sistemas públicos de educación, salud y seguridad social, entre otros. Así bien, el Estado a partir de 1960, habría ampliado su participación como espacio de las decisiones colectivas y en este sentido, habría extendido su condición de arena de lucha por las condiciones materiales de vida; la reducción de la política que debió operar el proyecto neoliberal en Ecuador fue relativamente menor que en otros casos nacionales.

Como botón de muestra, en esta etapa se dieron los primeros avances en el campo de la salud con la creación de un sistema nacional "subvencionado", centralizado en el Ministerio de Salud Pública, para saldar la fragmentaria oferta existente (Velasco, 1997: 27). En el sector educativo, los graves problemas de analfabetismo y baja cobertura dieron lugar a la Reforma Educativa de 1962 y su reformulación en 1973, tras el objetivo de universalizar la escuela primaria y formar cuadros técnicos para la incipiente industrialización del país (Ponce, 1997: 63). La seguridad social para esa época mostraba un desarrollo errático, entre otras cosas, por las características de un mercado de trabajo mayoritariamente informal, con pocas empresas formales con capacidad suficiente para crear una demanda de empleo. En ese contexto, una de sus características centrales fue la anexión de nuevas categorías de trabajadores bajo la lógica de un sistema fragmentado y con diferenciales prestaciones ${ }^{1}$ (SENPLADES, 2008:6).

Este limitado avance de acciones estatales no impidió que hacia 1980 se inicie la instrumentación de políticas de corte neoliberal. Luego de la muerte de Jaime Roldós en 1981, asumió la presidencia Oswaldo Hurtado (19821984), quien estableció el primer programa de "estabilización" de la economía encaminado a reducir el gasto público, controlar la inflación y mejorar los saldos de la balanza de pagos. Se trató de un período con fuerte concentración de la riqueza, principalmente por el proceso de "sucretización" de la deuda privada, la cual favoreció principalmente al sector bancario (Vos et ál., 2002: 11). Hacia 1984, bajo el gobierno del derechista León Febres Cordero (19841988), se amplía la agenda neoliberal con la apertura al mercado externo, la liberalización y desregulación de la economía y las finanzas, el desmonte de instrumentos ligados a la planificación estatal, la desactivación de la industrialización por sustitución de importaciones

$1 \quad$ Hacia 1980, el porcentaje de población protegida mediante el IESS y el Seguro Social Campesino era de 9,38\% del total de población; en 1985 de $12,6 \%$; $y$ hacia 1990 a $15,19 \%$ (SIISE). 
$y$ el énfasis en las exportaciones primarias. Lo interesante a resaltar, es que en esta primera fase del neoliberalismo se evidencia una particularidad que va a acompañar a todo este ciclo $y$ es que la aplicación de políticas neoliberales estará atada al devenir de las luchas intestinas de las alianzas socioeconómicas dominantes ${ }^{2}$. Como afirman algunos autores, no se trató de la búsqueda de vigencia plena y completa de las leyes del mercado. Más bien el "neoliberalismo a la ecuatoriana" supuso la reorientación del intervencionismo estatal para beneficiar a algunos empresarios con subsidios ligados a exportaciones $y$ aranceles a ciertas importaciones, control de precios para productos que antes habían sido desregulados y hasta controles en el mercado cambiario (Ramírez y Ramírez, 2005: 39). Se trató de una desregulación selectiva $y$ parcial evidenciando un proceso de "estatización del neoliberalismo" (Montúfar, 2000: 13). Es decir, resultará un neoliberalismo en donde la hegemonía de las alianzas a favor de políticas pro-mercado sin estar cuestionada tout court, suponga una lentificación y en algunos casos, límites a su avance. En el campo social, esto podrá observarse en los intentos por privatizar la seguridad social.

Con el socialdemócrata Rodrigo Borja (1988-1992) se esperaba una desactivación de aquel programa, pero se sostuvo una política de ajuste fiscal, se liberalizaron los precios de productos básicos de consumo y se dio paso a reformas estructurales mediante una ley de régimen tributario, sustentada en el fortalecimiento del impuesto al valor agregado y la reducción de las tasas marginales del impuesto a la renta (Vos et ál., 2002: 14) y nuevas normativas para el régimen arancelario y laboral.

Durante los 80, también los sectores de política social vieron modificado su anterior esquema. Por un lado, entre 1982 y 1984 se redujo la inversión pública en salud (Estrella y Crespo-Burgos, 2002: 236); en 1984 se anuló el reglamento que fijaba los precios de los medi-

$2 \quad$ Al histórico clivaje entre regiones (Sierra, Costa y Amazonía) se suman las diferencias entre elites económicas: empresarios ligados al comercio y/o intermediación y otros a la producción y exportación de materias primas. camentos, instrumento clave a fin de contener la privatización en esa área. También en esta década, comenzó a desarmarse el discurso universalista y a focalizarse la acción estatal en los grupos considerados "más vulnerables": niños y madres (Velasco, 1997: 28). Asimismo, cobraron fuerza las propuestas de "descentralización". En salud, se trató de "regionalizar" los servicios del Ministerio de Salud Pública, transfiriéndose responsabilidades hacia niveles subnacionales. En el campo de la educación, al tiempo que se proponía ampliar la cobertura, se producían sistemáticos recortes presupuestarios. Lo más relevante fue el desarrollo de programas y proyectos puntuales financiados por organismos internacionales que van a confirmar los nuevos principios de acción en este campo: la descentralización educativa, la focalización en sectores y territorios considerados "pobres", y el eje en la educación básica. En cuanto a la seguridad social, se consolida la lógica de instituir regímenes especiales, desincentivando la posibilidad de establecer un sistema de tipo universal. Al ya creado Seguro Social Campesino e Instituto Ecuatoriano de la Seguridad Social (IESS) se agregará el de las Fuerzas Armadas con su Instituto propio de Seguridad Social (ISSFA) y hacia el segundo lustro de los 90, le toca el turno a la Policía Nacional (ISSPOL).

En la esfera de la llamada "sociedad civil", bajo este contexto, se da la emergencia del Movimiento Indígena Ecuatoriano (MIE). Si bien la organización indígena registraba ya una larga tradición, en 1986 se produce la creación de la Confederación de Nacionalidades Indígenas del Ecuador (CONAIE). La siguiente década, con el funcionamiento de otras condiciones, dará inicio a una fase de alta conflictividad social que tendrá a este actor como protagonista.

\section{b) LA PROFUNDIZACIÓN DEL NEOLIBERALISMO (1990-2006)}

Existe acuerdo en sostener que es en la presidencia de Sixto Durán Ballén (1992-1996) cuando se consolida el modelo neoliberal. Se implanta un nuevo programa de "estabilización” que prioriza el ajuste fiscal. En forma complementaria, se instrumentan reformas 
que profundizan las ya propuestas en el sector financiero, agrícola y de hidrocarburos. Esto último para facilitar la inversión extranjera $y$ desregular los precios de los combustibles (Vos et ál., 2002: 15). Otras medidas fueron el aumento de las tarifas eléctricas y el alza de los derivados del petróleo, especialmente la gasolina. Asimismo, se plantea una ley de "Modernización del Estado" que tenía por objeto modificar el papel del Estado en la economía y sobre todo privatizar sus empresas y las áreas estratégicas. En este marco, se creó el Consejo Nacional de Modernización (CONAM) como organismo rector de los procesos de privatización (Vásconez, 2006: 367).

En el ámbito de la política social, se profundizó la transformación del papel del Estado. En 1993, se crea el Fondo de Inversión Social de Emergencia (FISE) para financiar proyectos de infraestructura demandados por poblaciones con mayores privaciones $y$ atender sus necesidades (Naranjo, 1997 y Vos, 2000). Este Fondo implicó el traslado de la ejecución de las acciones $y$ obras a empresas privadas $y / u$ organizaciones no gubernamentales, dando paso a una nueva modalidad de instrumentación de las intervenciones públicas (Naranjo, 1997: 98). De acuerdo con Vos et ál. (2002), justamente la invocación a los organismos no gubernamentales (ONG) e inclusive al sector privado empresarial para identificar, distribuir y ejecutar acciones en los grupos más vulnerables, formó parte del movimiento privatizador de la política social. En el área de salud, en 1992 se puso en ejecución el proyecto de Fortalecimiento y Ampliación de los Servicios Básicos de Salud (FASBASE) con financiamiento del BID, el cual planteó desarrollar sistemas locales de salud centrados en atención primaria y en 1994, se iniciaron las primeras pruebas piloto del cobro de servicios públicos de salud (Naranjo, 1997: 96). En el marco de la focalización, se creó el programa "Nuestros Niños" con financiamiento del BID, para la atención integral a menores de seis años en situación de pobreza. Su objetivo también fue buscar "una mayor participación de la sociedad civil en el financiamiento, ejecución y supervisión de las acciones del sector" (Vos, 2000: 140). En el campo educativo, también se consolidó la lógica de programas y proyectos con financiamiento externo, orientados a la descentralización educativa y la terciarización de servicios. Finalmente, respecto a la seguridad social, se propuso un nuevo sistema basado en la introducción de seguros privados — administradoras privadas de fondos (AFPs) la separación de las actividades de financiamiento de las de prestación de servicios y la recuperación de costos (Vos et ál., 2002: 16 y SENPLADES, 2008: 6).

En síntesis, en los 90 este enfoque político se propuso la desafectación del Estado en lo que hace a la garantía de las condiciones de vida de la población y profundizó la estrategia de traslado de estos asuntos hacia la sociedad civil qua mercado. Sin embargo, a pesar de estos avances en la agenda neoliberal, la relación en el interior del bloque hegemónico siguió siendo tensa. En esta fase, la "disputa faccional" entre las elites se concentró en torno a la orientación de las privatizaciones, el control de segmentos estratégicos del mercado (electricidad y telefonía) y por la privatización de la seguridad social (Ramírez, 2010: 19).

El conflicto social no se hizo esperar $y$ tuvo a los indígenas como protagonistas. Durante el primer lustro de los 90, giró en torno a reclamos sectoriales. Así, en el levantamiento de 1990, la protesta se centró en la denuncia por la concentración de la tenencia de la tierra, las políticas de riego, crédito y precios agrícolas y el reconocimiento oficial del carácter plurinacional del Estado. El discurso contestatario estuvo anclado a la demanda por reconocimiento a la dicotomía indígena-mestizo (blanco) y a los asuntos del mundo rural. En 1995, se creó la Coordinadora de Movimientos Sociales (CMS) y con ella se abrió la posibilidad de producir articulaciones políticas más amplias (respecto de la vertiente "indígena") dentro del bloque opositor. La solución del gobierno frente a ambas limitaciones (elitista $y$ popular) fue convocar a una consulta. Entre sus principales puntos se encontraba la reforma a la seguridad social y la desregulación laboral del sector público. El resultado fue una derrota para los intentos por profundizar las propuestas neoliberales. 
En los siguientes 10 años, ambas limitaciones se agravaron sin por eso verse cuestionado el neoliberalismo. En 1996, asumió Abdalá Bucaram, quien impuso un sistema monetario de convertibilidad, suprimió de subsidios y favoreció las reformas a la seguridad social, entre otras cuestiones. Esta agenda que permitió cierto consenso inicial entre el establishment, abrió frentes de conflicto (en el interior del empresariado y desde las organizaciones indígenas y los sindicatos públicos) que llevó a un desgaste vertiginoso de su legitimidad y a una prematura salida. Su sucesor, el titular del Congreso Nacional, Fabián Alarcón, mantuvo intacta esta política, aunque convocó a una Asamblea Constituyente que promulgó una nueva Carta Política en 1998. Esta dio vía libre a las propuestas privatizadoras, por ejemplo, eliminando la noción de áreas estratégicas y otras formas de propiedad estatal y comunitaria; pero también a la ampliación de algunos derechos, fundamentalmente civiles y políticos, por lo que habló de un "neoliberalismo con rostro social" (Ramírez, 2011: 58).

Una devastadora crisis se desató en 1999, bajo la presidencia de Jamil Mahuad (1998-2000) cuando se congelan los depósitos bancarios con consecuencias desbastadoras en la inversión, la actividad económica y el empleo. Otra medida restrictiva fue el retiro de los subsidios al gas natural, a la electricidad y combustibles, que afectó aún más el consumo de las clases populares. Como medida "compensatoria" se puso en marcha el programa focalizado de transferencias monetarias denominado "Bono Solidario"3. Asimismo, se aplicaron tarifas diferenciadas a la atención de la salud, según la categorización de los usuarios en grupos socioeconómicos, para la recuperación de costos. En el campo de la seguridad social, nuevamente en 1998 se encaró una reforma, hasta que por la Ley de Seguridad Social de 2001, se introdujo un sistema mixto de pensiones, con capitalización

$3 \quad$ El gobierno de Mahuad tomó como "colaborador estratégico" de la "sociedad civil" a la Iglesia, para que se encargara de receptar las solicitudes/inscripciones de los "pobres" (Recalde, 2007: 11). individual y solidaria. Este esquema fue impugnado por diversos sectores y se entabló una demanda por inconstitucionalidad que dejó sin efecto la participación de las administradoras.

El no pago de deuda externa y la corrida bancaria que ello desató, culminó con la dolarización de la economía, que despertó las protestas y movilizaciones sociales y el derrocamiento del Presidente en enero de 2000, conducida por una alianza que unió a la dirigencia indígena con sectores del mando militar. A pesar de esta novedad $y$ su fuerza democratizadora, el programa neoliberal continuó con el sucesor de Mahuad (su vice, Gustavo Noboa), quien mantuvo la dolarización y el paquete de medidas que beneficiaba al sector financiero. Pero además, elevó los precios de los combustibles $y$ del gas de uso doméstico. La conflictividad nuevamente se desató, estas medidas se retrotrajeron en abril de ese año; se diseñó un Plan Social de Emergencia, con el que adquirieron su completa institucionalización en la estructura estatal los denominados programas sociales "prioritarios"; es decir, un conjunto de acciones diseñadas bajo la lógica de la asistencia, la acción "mínima" o "básica" del Estado y la focalización.

Hacia el 2002, se inició la campaña por un nuevo periodo de gobierno $y$ entonces se restableció la alianza indígena-militar, que venció en los comicios, llevando a la presidencia al Cnel. Lucio Gutiérrez. Si bien, se esperaba el inicio del ciclo post ajuste, esto se derrumbó en los primeros meses de gestión. Se siguió avanzando en la agenda privatizadora, ahora con empresas públicas, pero la debilidad de los apoyos políticos del gobierno impidió su instrumentación.

El Bono Solidario pasó a denominarse Bono de Desarrollo Humano, al introducir la "corresponsabilidad" de los "beneficiarios" en relación con los componentes de educación y salud (básicamente certificar el acceso a la educación primaria y exigir controles de salud de niños y madres). Es decir, el programa se reconstruyó definiéndose ahora sí como de "atención a la pobreza" y bajo la lógica de que hay responsabilidades que deben asumir 
individualmente los que reciben el "beneficio". Asimismo, se redoblaron los esfuerzos para optimizar la focalización y depurar la base de beneficiarios del programa y se desarrolló un índice de bienestar para el país con base en cincuenta variables (SELBEN) (Recalde, 2007: 12 y 13).

Frente al abandono de los postulados de campaña, la alianza gubernamental se rompe. El resultado más rotundo fue el descenso del conflicto social y el debilitamiento de la capacidad de movilización de la alianza indígenamilitar, en la que ganó terreno el discurso más decididamente étnico. Su programa enfatizó la necesidad de retomar el proyecto de las autonomías territoriales $y$ de volver al trabajo de las "comunidades" (Ramírez, 2010: 26). Con esto no solo se perdía la posibilidad de alcanzar articulaciones pluralistas, sino inclusive se retrocedió y redujeron los planteos de cambio, ahora se trataba de recuperar otra comunidad perdida, no la ligada a algún proyecto integrador de sociedad.

Meses más tarde, se inicia el camino para un nuevo derrocamiento presidencial. En este caso encaminado por un conjunto de movilizaciones sin dirección política ni organizativa que pedían una nueva Asamblea Constituyente. Ningún movimiento organizado pudo liderar la protesta que dio fin al gobierno de Gutiérrez. En el nuevo escenario, asumió su vicepresidente, Alfredo Palacio. Prácticamente no hubo cambio en la agenda gubernamental, aunque se suspendió el contrato con la petrolera norteamericana oXY y se reformó la Ley de Hidrocarburos, mejorándose los ingresos fiscales, reorientados al cumplimiento de la agenda social. Esto fue impulsado por el Ministerio de Economía y Finanzas a cargo, hasta ese entonces, de un ignoto economista: Rafael Correa.

El relato hasta aquí expuesto da cuenta de las particularidades del caso ecuatoriano $y$ permite advertir la operatoria del componente neoconservador del neoliberalismo antes aludido. A continuación, se detallan algunas condiciones que permitieron iniciar la salida de este ciclo y complejidades a él asociadas.

\section{LA REVOLUCIÓN CIUDADANA: ENTRE EL RETORNO DEL ESTADO, LA "CRISIS \\ DE LA HEGEMONÍA NEOLIBERAL” Y LA TRANSICIÓN A LA SALIDA AL NEOLIBERALISMO}

\begin{abstract}
(...) una crisis no es un fenómeno meramente coyuntural, que provoca el abandono de determinadas creencias y la adopción de otras disponibles, sino que abre una "experiencia abismal", en la que toda inteligibilidad se quiebra. En la identificación de una crisis no es suficiente verificar la dislocación objetiva de un determinado horizonte de pensamiento, lo que deja abiertas las puertas a su abandono (...). La crisis tiene así también un componente subjetivo que se pone de manifiesto cuando se admite que una tradición está históricamente (objetivamente) deshecha pero aún se persiste en ella, puesto que ninguna otra aparece como más aceptable o menos problemática. Una crisis, en definitiva, instala un terreno inhabitable, pero tampoco es traspasable
\end{abstract}

Elías Palti (2002: 15).

Como afirman Danani y Grassi (2008), lo que comúnmente se señala como "crisis neoliberal" exige algunas distinciones. Así, una "crisis de la hegemonía neoliberal" no necesariamente involucra una "crisis del neoliberalismo" y menos aún, su completo abandono como marco referencial en el sentido de la cita con la que se abre este apartado. Desde este lugar es posible sostener que hacia principios de 2007 , cuando se produce la llegada de Rafael Correa al poder, se confirma la crisis de la hegemonía neoliberal, en tanto se develan los límites en la capacidad de dirigir y dominar, que habían alcanzado ciertas alianzas socioeconómicas y políticas para sostener políticas neoliberales; también límites para producir reconocimiento social para la orientación impresa a tales políticas e imponer y presentar los intereses propios como el interés general (Danani y Grassi, 2008: 260). El hecho de que Rafael Correa llegue al poder sin postular candidatos para el Congreso Nacional, no es un dato menor. Bajo un discurso 
abiertamente en contra de los partidos políticos que habían "traicionado" una y otra vez sus promesas de campaña, se hacía referencia a la envergadura que adquiría la crisis de hegemonía. El discurso de Correa planteó la necesidad de ir directamente a una Asamblea Nacional Constituyente como primer paso para una salida al ciclo neoliberal.

No obstante, diversos elementos permiten ir más allá en este diagnóstico de la crisis del neoliberalismo, como es contramarcha al proceso de supresión de la política. Esto pudo verse en algunos contenidos del nuevo texto constitucional como en un conjunto de políticas. En primer término, la nueva Carta Política estableció un nuevo patrón de relaciones entre Estado, mercado y sociedad. El Estado adquiere un nuevo estatus: de "Estado legal de derecho" a "Estado constitucional de derechos y justicia". Ligado a ello, una nueva concepción de los derechos marcadamente social y garantista, la ampliación y ejecutabilidad de los mismos mediante una institucionalidad con rango constitucional $^{4}$ y la dominancia de una lógica de universalización de los derechos, frente a la mirada segmentadora y particularista de la asistencia y la focalización. Sumado a ello, la incorporación de la noción de bienestar colectivo respetando estilos de vida individual vs. la más restrictiva consecución del "bienestar individual”. Del mismo modo, artículos que proponen articulaciones de lo socio-económico frente a la escisión de lo económico y lo social, tan característica del anterior ciclo. Reconexión basada en una manera alternativa de concebir el desarrollo, ahora como medio para alcanzar el Buen Vivir (lo que supone poner a la vida como centro de todas las acciones humanas) y no como fin en sí mismo o como sinónimo de

$4 \quad$ Se establecen dos regímenes que tienen por finalidad garantizar el cumplimiento de lo establecido en el texto constitucional. Por un lado, el Régimen de Desarrollo y por el otro, el Régimen del Buen Vivir. Este último supone una institucionalidad (Sistema de Inclusión Económica y Equidad Social), en tanto conjunto articulado y coordinado de sistemas, políticas, instituciones, normas, programas y servicios, que aseguran el ejercicio, garantía y exigibilidad de los derechos (ver Ramírez Gallego en este volumen). crecimiento económico. Finalmente, la recuperación y el fortalecimiento de lo público estatal, lo que incluye una renovada manera de entender su gestión ("integralmente descentralizada $y$ desconcentrada" vs. gestión estatal centralizada y vs. descentralizada como sinónimo de privatizadora).

Paralelamente $y$ con posterioridad a este marco normativo, se llevaron a cabo políticas y se sancionaron leyes que concretaron aún más este trastocamiento. Muchas de ellas tuvieron como objetivo la re-afectación del Estado como ámbito privilegiado de las decisiones colectivas. Es interesante remarcar que tal reintroducción se hizo bajo un discurso de representación del interés general y vocación universalista ${ }^{5}$, con el objetivo de "separar al Estado de los poderes fácticos" que lo habían parcializado y privatizado. El robustecimiento de los factores de poder estatal va a transformar sus políticas en la arena de lucha por las condiciones materiales de vida. Por ello, se habla que en este ciclo "la sociedad y la nación hacen uso de su Estado como agente fundamental de una estrategia de desarrollo endógeno y soberano" (Ramírez, 2011: 24. Cursiva del autor). Entre las acciones que confirmaron la reinstalación de la política en el ámbito estatal se destacan las siguientes:

1) Recuperación de la planificación estatal a través de la Secretaría Nacional de Planificación y Desarrollo (SENPLADES), en lugar del Consejo Nacional de Modernización (CONAM).

2) Una definición alternativa de "descentralización" y "desconcentración" de funciones del Estado, extendiendo su "llegada" a todo el territorio nacional.

5 Una serie de slogans oficiales expresan este cambio: "La patria ya es de todos" o "Volver a tener patria”, planteó la posibilidad de recuperar ese sentido de nosotros (más allá de cualquier clivaje étnico, de clase, religioso, etc.), de interés colectivo y compartido ligado a un proyecto de integración. En este andamiaje toma también significado la idea de una "Revolución Ciudadana", que hace eje en una subjetividad integradora: ciudadanas y ciudadanos activos y empoderados. 
3) Recuperación de la soberanía nacional en sectores estratégicos, mediante la generación y fortalecimiento de empresas públicas (petróleo, minería, servicios públicos, etc.) y mixtas (públicas-privadas o públicas integradas con dos o más Estados nacionales).

4) Prioridad a los capitales nacionales $y$ al ahorro interno como medios para promover el desarrollo endógeno. Por ejemplo, recursos colocados en el Instituto Ecuatoriano de Seguridad Social - se creó el Banco del Afiliado (BIESS) - fueron direccionados al financiamiento de proyectos nacionales estratégicos (centrales hidroeléctricas, refinerías, infraestructura vial básica, etc.).

5) Reforma tributaria que incrementó la eficiencia y progresividad, aumentando la presión sobre los sectores más acomodados.

6) Renegociación de contratos del Estado con empresas transnacionales (especialmente en la explotación de petróleo), aumentado la participación estatal y los aportes de esta renta en el presupuesto nacional.

7) Regulación del sector bancario financiero y recuperación de la banca pública de desarrollo mediante líneas de créditos a pequeños $y$ medianos sectores productivos. Separación de la banca de los medios de comunicación $y$ de otras empresas, $y$ de los bancos de los fondos de pensión y de las aseguradoras.

8) Eliminación de la autonomía del Banco Central.

9) Reducción del peso de la deuda externa en el presupuesto público y aumento del gasto social (esto supuso el análisis $y$ revisión de aquella considerada ilegítima, su judicialización y su renegociación).

10) Impulso de políticas redistributivas de corte universal, a fin de atacar el problema de la desigualdad y efectivizar derechos consagrados en la norma constitucional $^{6}$.

$6 \quad$ Estas políticas tuvieron efecto directo en la reducción de la desigualdad (entre 2006 y 2010 bajó $6,7 \%)$. También en otros indicadores sociales, tales como pobreza por NBI (entre 2005 y 2009
Ahora bien, esta recuperación de la política que supuso una reconfiguración del Estado $y$ un re-traslado de asuntos desde el ámbito social-mercantil al ámbito público estatal, no estuvo exenta de contradicciones e inercias que mostraron el nivel de hegemonía que logró alcanzar el neoliberalismo en Ecuador. Un ejemplo claro puede verse en el campo de las políticas sociales.

En primer término, en el ámbito de la educación pública hubo avances importantes en la eliminación de barreras de acceso. Por ejemplo, se suprimió el cobro de matrículas, se amplió la distribución gratuita de textos, uniformes escolares y alimentación escolar. Asociado a ello, se amplió la oferta educativa oficial mediante la extensión de la jornada laboral de los maestros a 8 hs., hubo aumentos en cargos docentes y de los salarios. En el campo de la salud pública, se avanzó en la gratuidad $y$ ampliación del servicio de atención primaria, medicamentos gratuitos $y$ atención domiciliaria en barrios populares ${ }^{7}$. Asimismo, se estableció una jornada laboral extendida para el personal médico y asistente. Todo esto, en ambos casos, en procura de ampliar la cobertura y democratizar el acceso, rezagos históricos de ambos sectores (no solo en términos de cantidad, sino ampliar el ingreso de sectores que nunca antes o en pequeña proporción se habían incorporado) ${ }^{8}$. También se sancionó la gratuidad de la educación pública universitaria hasta tercer nivel, que hasta el año 2008,

bajó 6\%), extrema pobreza por NBI (disminuyó casi $5 \%$ entre 2005 y 2009), pobreza por ingreso (de $37,62 \%$ en 2006 pasó a $32,76 \%$ en 2010 ) y extrema pobreza por ingreso (pasó de $16,89 \%$ en 2006 a 13,09\% en 2010) (Fuente: INEC-ENEMDUR/ SENPLADES, 2011).

$7 \quad$ Así, la tasa de consultas del Ministerio de Salud Pública por cada 100 mil habitantes, pasando de 95 en el año 2006 a 243 en el 2010 (Fuente: MSP/ SENPLADES, 2011).

8 En 2001, la tasa neta de escolarización primaria ascendía a $90,2 \%$ y la secundaria a $44,9 \%$; en el 2009, la tasa neta de matriculación en la Enseñanza General Básica fue de 93,5\% y en el bachillerato de $54,8 \%$ (Fuente: SIISE a partir de los Censos de Población y Vivienda. Para el 2009, datos del Ministerio de Educación del Ecuador). 
estaba arancelada ${ }^{9}$. Empero, estos esfuerzos concentrados en la ampliación de cobertura tuvieron efectos de implosión al interior de los servicios que no estaban preparados para atender el crecimiento de la demanda, generando problemas serios de eficiencia y efectividad. Por otra parte, al igual que en el periodo neoliberal, la calidad siguió siendo el gran ausente de esta extensión, cuestionando gravemente los términos en los se desarrolla "la disputa por las condiciones de vida".

Asimismo, han existido esfuerzos para instrumentar la articulación entre las políticas económicas y sociales, quizás uno de los elementos más innovadores ha sido el impulso a la economía social y solidaria. Se crearon el Ministerio de Inclusión Económica y Social (que suplantó al de Bienestar Social) y dentro de este, el Instituto de Economía Popular y Social. En mayo de 2011, se aprobó la Ley Orgánica de la Economía Popular y Solidaria $y$ del Sector Financiero Popular y Solidario, $y$ mediante esta, se creó la Corporación Nacional de Finanzas Populares para atender a este segmento de la economía. Sumado a ello, se desarrollaron una serie de programas que se definen como de "inclusión socioeconómica" (ej. Programa de Alimentación Escolar (PAE) e Hilando el Desarrollo) a través de los cuales, artesanos o pequeños productores agrícolas amplían su campo de acción al incorporarse al sistema de compras públicas. El mayor obstáculo reside, más allá de la declaración formal, en una visión residual de este campo de acción, ligada a los "márgenes del capitalismo" $y$ a intervenciones para sectores en condiciones socioeconómicas precarias.

Por otra parte, si bien el gobierno ha diseñado estos y otros programas que inicialmente podrían considerarse alternativos a los típicos de la etapa neoliberal, sigue siendo destacada la importancia que tiene en la agenda gubernamental el Bono de Desarrollo

$9 \quad$ La tasa neta de matrícula en el nivel superior pasó de $22,9 \%$ en 2006 a 28,3\% en 2010 . El crecimiento fue más significativo en el $40 \%$ más pobre de la población: la matricula pasó de $8,2 \%$ a $15,6 \%$ respectivamente (INEC-ENEMDUR/ SENPLADES, 2001).
Humano $^{10}$. En este sentido, si bien amplió el rango de beneficiarios ${ }^{11}$ y el monto del beneficio $^{12}$, se incorporaron elementos que mejoran las posibilidades de "autovalía" (crédito asociado al Bono), no se desarmó la lógica selectiva pro-pobre y asistencial típica de los programas de transferencias monetarias condicionadas. Asimismo, llama la atención la falta de articulación entre este tipo de programas $y$ acciones que refuercen el sentido de universalidad, la incorporación efectiva a la sociedad y la "responsabilidad colectiva"; por ejemplo, mediante la conexión con la seguridad social, esto daría real contenido "solidario" al sistema, más allá del aporte contributivo o no contributivo de sus afiliados ${ }^{13}$.

Pero quizás lo que demuestra de manera rotunda las dificultades para desarmar el andamiaje neoliberal, es la misma institucionalidad del Ministerio de Inclusión Económica y Social, que a pesar del cambio de nombre, sigue siendo pensado como ente encargado de los programas y proyectos ligados a la atención de población vulnerable y en estado de pobreza ${ }^{14}$. Por ejemplo, desde

10 En la actualidad, el Bono de Desarrollo Humano es el programa social de mayor alcance del país con una cobertura de 1200000 personas y es además, el referente para determinar la elegibilidad focalizada a otros programas (por ejemplo, Programa Aliméntate Ecuador).

11 De manera gradual se incorporaron también adultos mayores y discapacitados. Por las mejoras en la focalización se logró cubrir al 95\% de los hogares bajo la línea de pobreza (MCDS, 2010).

12 Inicialmente el Bono suponía una transferencia de 15 dólares mensuales. En el año 2007, aumentó a 30 dólares y en el 2009, a los 35 dólares. Asimismo, se incorporaron otros beneficios para los adultos mayores como la pensión asistencial y jubilar; sin embargo, esta no está integrada al IESS.

13 El Estado subsidia el $40 \%$ de las pensiones jubilares en el caso del IESS y el $60 \%$ en el caso de los seguros para las Fuerzas Armadas y la Policía Nacional. Sin embargo, en el caso del IESS, el sistema beneficia principalmente a los grupos más ricos.

14 Baste como ejemplo el discurso oficial del Ministerio: "El MIES es ese puente para que nuestra población ecuatoriana (las madres del Bono, los campesinos pobres, los emprendedores populares, las asociaciones y cooperativas, nuestros niños 
el Instituto de la Niñez y la Familia (INFA) ${ }^{15}$ dependiente de este Ministerio se sigue atendiendo al "desarrollo infantil" bajo la idea de la "niñez", en tanto, grupo vulnerable. De hecho, no es un dato menor que desde esta entidad se maneja lo relativo a la educación inicial y no desde el Ministerio de Educación.

En el campo de la seguridad social, se ha producido un significativo aumento en la cobertura $^{16}$ (mediante la inclusión de aportes "voluntarios", fijando la obligatoriedad en la afiliación de las empleadas domésticas y recientemente, volviendo "delito" la no afiliación al IESS para cualquier trabajador en relación de dependencia). Empero no se ha avanzado en una reforma integral del sistema. Por un lado, sigue funcionando como un campo fragmentado institucional y prestacionalmente y por ende, en términos de reconocimientos, derechos y subjetividades. No hay articulación entre el IESS, el ISFA, el ISPOL y el Seguro Social Campesino a fin de universalizar los servicios. Tampoco en lo relativo a la tención de la salud se integraron tales instituciones a un único sistema nacional de salud. La seguridad social solo cubre a los afiliados y no se extiende a los grupos familiares. Tampoco está plenamente cubierto el sector formal ${ }^{17}$ de la economía. En cuanto al informal18, aún mayori-

$y$ niñas, los jóvenes, nuestros abuelitos y nuestra población con capacidades especiales)". En: $<$ http://www.mies.gov.ec/index.php/institucional/ historia $>$

15 Esto no resta importancia a algunos avances en este campo, por ejemplo, la institucionalización de un INFA de carácter público. Esto supuso la desaparición de cuatro programas que funcionaban de manera descoordinada $y$ fragmentada y especialmente, la desaparición del INNFA de carácter privado (Instituto Nacional de la Niñez y la Familia).

16 En diciembre de 2010, el 55,1\% de los ocupados plenos estaban afiliados al IESS, frente al 39,2\% de registrados en el año 2007 (INEC-ENEMDUR/ SENPLADES, 2011).

17 Del sector formal, el 26,5\% no tiene afiliación al IESS (ENEMDU, 2010).

18 Solo el $12 \%$ tiene afiliación al IESS (ENEMDU, 2010). tario ${ }^{19}$ en Ecuador, los rezagos siguen siendo enormes.

Respecto al tema laboral, lo más importante fue la eliminación mediante Mandato Constitucional de la tercerización. En el campo de los trabajadores públicos, resultó un avance la decisión de aglutinar a todos bajo una misma Ley Orgánica del Servicio Público y por ende, una misma política salarial y la eliminación de "privilegios", especialmente en sectores altamente corporativizados como petróleo, Banco Central, militares y policías. Esto permitió redefinir la burocracia estatal bajo términos de mayor igualdad. Sin embargo, en el sector privado prácticamente no se ha avanzado, salvo los efectos de las tres alzas decretadas en el ingreso básico de los trabajadores entre 2009 y 2011. Asimismo, hasta la fecha no existe ningún tipo de seguro de desempleo ni sistema de asignación familiar.

Uno de los grandes avances se ha producido en el tratamiento de los discapacitados. Actualmente, se está llevando a cabo el programa "Manuela Espejo" que incluye un barrido censal (mediante brigadas), a fin de identificar las causas de las discapacidades, conocer la realidad de esta población y desde el terreno, identificar personas que requieran atención. Es importante indicar que esta metodología ha permitido llegar inclusive a personas que por su discapacidad y estado de abandono nunca antes habían recibido atención. Por su parte y asociado a este programa, se inauguró la "Misión Joaquín Gallegos Lara”, la cual incluye la identificación de un responsable de los cuidados al discapacitado y la recepción de un bono mensual, además de medicinas y capacitación. El punto es que este programa se desarrolla bajo una serie de conceptualizaciones ligadas a la más clásica asistencia. También como un esfuerzo puntual desde la Vicepresidencia de la República sin conexión con el resto de acciones estatales y sobre todo, articulación con políticas de orientación universal como educación, salud y seguridad social.

19 La escasa cobertura también se explica por el tamaño del sector informal. Se estima que actualmente la actividad informal alcanza el $47 \%$ de la PEA. Los servicios domésticos alcanzan el 3,4\% de la PEA (ENEMDU, 2010). 
Desde el lado de la sociedad civil, hay que indicar que el traslado de la política al Estado no desactivó los espacios "societales" en los que esta intentó marginalmente mantenerse activa durante el ciclo precedente. Empero, bajo el nuevo escenario iniciado en 2007, se develó hasta qué punto este campo resultó también profundamente penetrado por una dinámica particularista y privatizante. Al poco tiempo de aprobado el texto constitucional, la ambivalente relación que tuvo el movimiento indígena con el gobierno correista se transformó en frontal oposición bajo un discurso étnico y ahora decididamente antiestatal. En marzo de 2010, se reunieron los dirigentes de la CONAIE y la Junta Cívica de Guayaquil, reducto de los intereses oligárquicos de la Costa, con el fin de sumar fuerzas y apoyar un frente opositor al gobierno. Más allá de las diferentes voces de rechazo que generó tal acercamiento, muchos sostuvieron que entre ambos actores existía un "punto nodal de encuentro" ligado a la demanda por la autonomía y el derecho al auto-gobierno (Ramírez, 2010: 33). Lo interesante de esta lectura, que sobrepasa cualquier análisis en clave "indígena" del asunto, da cuenta de un punto de convergencia ligado a una posición particularista $y$ contraestatal que se remonta al periodo neoliberal. Una posición que devela a su vez la diversidad de nociones de "comunidad" (en tanto proyecto de sociedad) en disputa que se abrieron en esta fase.

Desde este breve punteo queda clara la dificultad para sostener que se está frente a una incuestionable superación del neoliberalismo. Como indican Danani y Grassi, este supuso algo más que "una política y/o modelo económico". Se constituyó, por el contrario, en "una ideología, en el sentido de representación del mundo y de acciones en pos de su realización" (2008: 260). De esta forma, en el nuevo ciclo inaugurado a partir de la llegada al poder de Rafael Correa, puede hablarse de que el neoliberalismo perdió la condición de "representación única (como única vida posible)", más no su "capacidad de disputa por su imposición y por la realización de tales intereses por parte de actores concretos" (2008: 260). En definitiva, lo señalado es indicador de la complejidad involucrada en la descomposición del ciclo neoliberal. No solo se trata de la pervivencia de "políticas neoliberales", sino elementos que expresan la vigencia de ese componente neoconservador del neoliberalismo detallado en las secciones precedentes. Esto, más que "fracasos" o "derrotas", refieren a las dificultades que involucra recorrer, en los términos de Palti, la experiencia de la transición entre un "horizonte de pensamiento" que está en proceso de fenecimiento pero que sigue disputando y otro que está intentando nacer, que se gesta (en el sentido en que se va produciendo) en su cotidiana dinámica de confrontación con aquel.

\section{CONSIDERACIONES FINALES}

El artículo buscó problematizar un aspecto del proyecto neoliberal en un caso concreto, Ecuador, en un campo de intervenciones estatales específicas: las políticas sociales. Este aspecto supuso la reducción de la política y su traslado al ámbito de la sociedad civil. En esta operatoria se buscó, a su vez, transformar ese ámbito social en un orden mercantil donde la producción y reproducción de la vida de las personas debía inscribirse en los estrictos límites de la responsabilidad individual.

El retorno del Estado y el re-traslado de la política a sus espacios que se observa a partir de 2007, en este país, si bien desandó parte de aquella operatoria, muestra límites ligados al elemento más estructural del cambio operado durante el neoliberalismo: la des-socialización de la vida. Así, si bien, hay indicios claros que con el retorno del actor estatal se da una ampliación de las bases contingentes (disputables) de la definición de las condiciones materiales de vida de la sociedad, esto no necesariamente supone avances en una re-socialización en clave de solidaridad y "responsabilidad social". El proceso de individualización, de desarticulación y desigualación social que involucró el neoliberalismo todavía no parece profundamente cuestionado. Asimismo, la re-afectación del Estado en las decisiones colectivas ha implicado introducir la conflictividad social en su territorio, que más allá del discurso y la pretensión de representar el interés general, todavía resulta opaco el mapa de intereses en juego, 
las concepciones de cambio que están involucradas en sus principales actores y los márgenes reales de acción que detentan. Así, más allá de las innegables transformaciones involucradas en el proyecto de la "Revolución Ciudadana", las dificultades que persisten hablan de la necesidad de repensar los más soterrados constructos del neoliberalismo, si es que se quiere tener algún éxito en su superación.

\section{BIBLIOGRAFÍA}

LIBROS

Danani, Claudia y Beccaria, Alejandra. "Sistema de Seguridad Social y mercado de trabajo: evolución de la cobertura en la Argentina entre 1990 y 2010". Protecciones y desprotecciones: la seguridad social en la Argentina (19902010). Danani, Claudia y Hintze, Susana (coords.). Bueno Aires. Universidad Nacional de General Sarmiento, 2011: 103-151.

Danani, Claudia y Grassi, Estela. "Ni error, ni omisión. El papel de la política de Estado en la producción de las condiciones de vida $y$ de trabajo. El caso del sistema previsional de la Argentina (19932008)". Trabajo, ingresos y políticas en Argentina. Contribuciones para pensar el siglo XXI. Lindenboim, Javier (comp.). Buenos Aires. EUDEBA, 2008: 259-298.

Danani, Claudia y Hintze, Susana. "Introducción. Protección y seguridad social para distintas categorías de trabajadores: definiciones conceptuales, propuestas de abordaje e intento de interpretación". Protecciones y desprotecciones: la seguridad social en la Argentina (1990-2010). Danani, Claudia y Hintze, Susana (coords.). Buenos Aires. Universidad Nacional de General Sarmiento, 2011: 9-29.

Estrella, Eduardo y Crespo-Burgos, Antonio. "El Ministerio de Salud Pública y la OPS/OMS en el Ecuador". El cóndor, la serpiente y el colibrí. La OPS/OMS y la salud pública en el Ecuador en el siglo XX. Quito. OPS, 2002: 229-241.
Lechner, Norbert. "El proyecto neoconservador $y$ la democracia". Autoritarismo y alternativas populares en América Latina. Rojas Aravena, Francisco (ed.). Costa Rica. Flacso, 1982: 23-70.

Ministerio Coordinador de Desarrollo Social. Programas Sociales 2010. Quito: MCDS, 2010.

Montúfar, César. La reconstrucción neoliberal. Febres Cordero o la estatización del neoliberalismo en el Ecuador 1984-1988. Quito: Abya Ayala, 2000.

Naranjo, Mariana. "Las políticas sociales: ¿un medio para desarrollar el capital humano?". Pobreza y Capital Humano. Quito: Secretaría Técnica del Frente Social (STFS), 1997: 87-130.

Offe, Claus. "Los nuevos movimientos sociales cuestionan los límites de la política institucional". Partidos políticos y nuevos movimientos sociales. Madrid. Editorial Sistema, 1996: 163-244.

Ponce, Juan. "La Educación: a la espera de la equidad y la calidad". Pobreza y Capital Humano. Quito. Secretaría Técnica del Frente Social (STFS), 1997: 59-86.

Ramírez Gallegos, Franklin "El Estado en disputa: Ecuador 1990-2011". El Estado de los Derechos de la Niñez y Adolescencia, a 21 años de la Convención de los Derechos del Niño. En prensa. Quito: ODNA, Save the Children, Plan Internacional $y$ UNICEF.

Ramírez Gallegos, Franklin y Ramírez Gallegos, Jacques Paul. La estampida migratoria ecuatoriana. Crisis, redes internacionales y repertorios de acción migratoria. Quito: UNESCO, Abya Ayala y CIUDAD-Centro de Investigaciones, 2005.

Recalde, Paulina. La redimensión de la Politica Social en el Ecuador: el Bono de Desarrollo Humano en el gobierno de Rafael Correa. Quito: Observatorio Político del Centro de Estudios Latinoamericanos, Pontificia Universidad Católica del Ecuador, 2007.

Secretaría Nacional de Planificación y Desarrollo. "Propuesta de Reforma al 
Sistema de Pensiones del Ecuador". Policy Paper. Quito. SEnPlades, 2008: 1-54.

Secretaría Nacional de Planificación y Desarrollo. 100 Logros de la Revolución Ciudadana. Quito: SENPLADES, 2011.

Vásconez, Allison; Córdoba, Rossana y Muñoz, Pabel. "Políticas sociales en el Ecuador en el período 1978-2003. Sentidos, contextos, y resultados". Política y políticas públicas en los procesos de reforma de América Latina. Franco, Rolando y Lanzaro, Jorge (Eds.). México. CEPAL y Flacso México, 2006: 353-383.

Velasco, Margarita. "El reto de la salud para Todos". Pobreza y Capital Humano. Quito: Secretaría Técnica del Frente Social (STFS), 1997: 23-58.

Velasco, Margarita. "La salud de la población: medio siglo de cambios". Estado del País. Informe cero. Ecuador 19502010. Quito. Flacso, Contrato Social por la Educación, ESPOL, PUCE, ODNA, Universidad de Cuenca y UNICEF, 2011: 323-342.

Vos, Rob. Ecuador 1999. Crisis económica y protección social. Quito: Frente Social, Sistema Integrado de Indicadores Sociales del Ecuador (SIISE) y Abya Ayala, 2000.
Vos, Rob; León, Mauricio y Ramírez Gallegos, René. Informe nro. 1. Política Social y tendencias en el gasto social: Ecuador 1970-2002. Quito: Institute of Social Studies (ISS), Frente Social del Ecuador y Sistema Integrado de Indicadores Sociales del Ecuador (SIISE), 2002.

REVISTAS

Palti, Elías. "Extraña pareja: marxismo y posmodernismo. Acerca de Los orígenes de la posmodernidad de Perry Anderson". Revista Punto de Vista 25 (74). Buenos Aires. Siglo XXI, 2002: 15-21.

Ramírez Gallegos, Franklin. "Fragmentación, reflujo $y$ desconcierto. Movimientos sociales y cambio político en el Ecuador (2000-2010)". Observatorio Social de América Latina 11 (28). Buenos Aires. CLACSO, 2010: 17-48.

Ramírez Gallegos, Franklin y Minteguiaga, Analía. "El nuevo tiempo del Estado. La política posneoliberal del correismo". Observatorio Social de América Latina 8 (22). Buenos Aires. CLACSO, 2007: 87-103.

Fecha de ingreso: 29/11/2011 Fecha de aprobación: 10/02/2012 